\title{
Absolute Stability of 3-DOF Bilateral Haptic Systems
}

\author{
Jian Li ${ }^{*, * *}$ Mahdi Tavakoli** Qi Huang ${ }^{* * *}$ \\ * School of Energy Science and Engineering, University of Electronic \\ Science and Technology of China, Chengdu, Sichuan, China 611731 \\ (e-mail: uajian@gmail.com. \\ ** Department of Electrical and Computer Engineering, University of \\ Alberta, Edmonton, AB T6G 2V4, Canada (e-mail: \\ mahdi.tavakoli@ualberta.ca) \\ *** School of Energy Science and Engineering, University of Electronic \\ Science and Technology of China, Chengdu, Sichuan, China 611731 \\ (e-mail: huangqi@uestc.edu.cn)
}

\begin{abstract}
:
A 3-degrees-of-freedom (DOF) bilateral haptic system can be modeled as an 2-port network where each port connects to a termination defined by 3 inputs and 3 outputs. The stability analysis of such systems is not trivial due to dynamic coupling across the different DOFs of the robots, the human operators, and the physical/virtual environments, and unknown dynamics of the human operators and the environments exacerbate the problem.

Llewellyn's criterion only allows for absolute stability analysis of 1-DOF bilateral haptic systems, which can be modeled as 2-port networks. The absolute stability of a general 3-DOF bilateral haptic system cannot be obtained from the applications of Llewellyn's criterion to each DOF of the bilateral system. In this paper, we present a straightforward and convenient criterion for absolute stability analysis of a class of 3-DOF bilateral haptic systems. As a case studies, a 3-DOF bilateral haptic system is studied for absolute stability with simulations confirming the theoretical stability conditions.
\end{abstract}

Keywords: three-port network, bilateral haptic system, absolute stability.

\section{INTRODUCTION}

Haptic systems have recently found applications in telemedicine, robotic-assisted surgery and therapy systems. A 3 -DOF bilateral haptic system can be modeled as an 2port network where each port (terminal) connects to a 3 DOF termination. For a teleoperation system consisting of a teleoperator comprised of master(s), slave(s) and controllers coupled to terminations consisting of human operator(s) and environment(s), closed-loop stability is critical for safe and effective teleoperation. Investigation of teleoperation system stability using common closed-loop stability analysis tools in the control systems literature is not possible because the models of the human operator(s) and the environment(s) are usually unknown, uncertain, and/or time-varying. However, research has shown that it is still possible to draw stability conditions for a haptic teleoperation system under unknown "terminations" as long as they are passive. These stability conditions can be categorized as passivity and absolute stability criteria.

For stability analysis of 1-DOF $n$-lateral haptic systems, passivity is used in Raisbeck (1954) for $n=2$, in Shahbazi et al. (2010); Panzirsch et al. (2012) for $n=3$, and in Mendez and Tavakoli (2010) for any $n \geq 2$. Specifically, in Raisbeck (1954), Raisbeck's method is useful as a passivity criterion for 1-DOF bilateral teleoperation systems based on the immitance matrix of the teleoperator. Shahbazi et al. (2010) performed stability analysis for a dual-user (trilateral) teleoperation system based on the passivity definition for a three-port network. In (Panzirsch et al. (2012)), Panzirsch et al. proposed a time-domain passivity observer/passivity controller approach for a dual-user (trilateral) teleoperation system. Mendez and Tavakoli (2010) presented a criterion (necessary and sufficient) for passivity of general $n$-port networks, which can model 1-DOF $n$-lateral haptic systems.

Passivity of a multi-port network is a conservative condition for its coupled stability. A less conservative condition, absolute stability is discussed in Haykin (1970); Llewellyn (1952) for $n=2$, in (Khademian and Hashtrudi-Zaad (2011); Li et al. (2013a)) for $n=3$, and in $\mathrm{Ku}$ (1963) for any $n \geq 2$. Specifically, Llewellyn (1952) proposed an absolute stability criterion for two-port networks, which model 1-DOF bilateral teleoperation systems, based on the immitance matrix of network (the teleoperator). Khademian and Hashtrudi-Zaad (2011) analyzed absolute stability of a dual-user (trilateral) teleoperation system by reducing the three-port network to an equivalent two-port network, paving the way for the applications of Llewellyn's criterion. $\mathrm{Li}$ et al. in ( $\mathrm{Li}$ et al. $(2013 \mathrm{a}, \mathrm{b})$ ) presented an absolute stability criterion for a class of trilateral haptic systems. In (Ku (1963)), $\mathrm{Ku}$ studied $n$-port network stability if the impedance matrix of the $n$-port network conforms to 


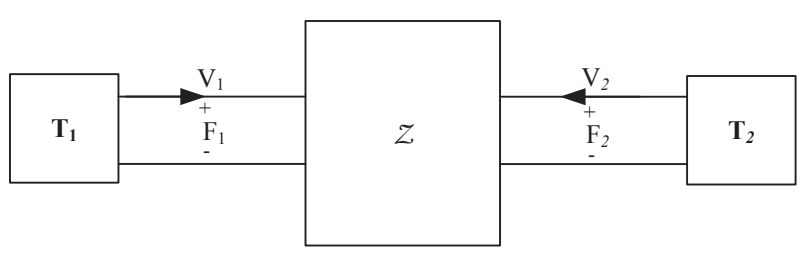

Fig. 1. An 2-port network where each port (terminal) connects to an $m$-DOF termination.

the tri-diagonal Jacobian form. The above research only addresses the absolute stability analysis of 1-DOF haptic teleoperators.

In past research, for stability analysis of multi-DOF bilateral (Speich and Goldfarb (2005)) and tri-lateral haptic systems (Malysz and Sirouspour (2011)), the multi-DOF systems are decoupled to $1-\mathrm{DOF}$ systems. Then, various stability criteria for 1 -DOF $n$-lateral haptic systems are used. This poses difficulties in terms of decoupling a coupled haptic system especially because the human operator(s) and the environment(s) terminations are also coupled themselves. In this paper, we present a criterion to analyze the absolute stability of 3-DOF bilateral haptic systems directly and without a need for decoupling. As a case study, we consider a 3-DOF bilateral haptic system and use the proposed absolute stability criterion to design stabilizing controllers for the system.

The rest of the paper is organized as follows: The next section introduces simple motivating examples to show that the absolute stability criteria for 1-DOF bilateral haptic systems fail to analyze the absolute stability of 3-DOF bilateral haptic systems. Section 3 gives mathematical definitions and lemmas for analysis of absolute stability. Next, in Section 4, the proposed absolute stability criterion for 3 -DOF bilateral networks is derived. Then, as a case study to show how the resulting absolute stability criterion can be utilized, in Section 5, a 3-DOF bilateral teleoperation system with position-position control is considered, the absolute stability conditions in terms of system parameters including controller gains are found, and simulations to verify the validity of the calculated absolute stability conditions are presented. Section 6 contains concluding remarks.

\section{MOTIVATION}

Llewellyn's criterion has been used to analyze the absolute stability of 1-DOF bilateral teleoperation systems. In the following, using one example, we show why it cannot be used for coupled 3-DOF bilateral teleoperation system. For absolute stability, both terminations of the two-port network need to be passive. For a coupled 3-DOF bilateral teleoperation system, consider the following termination for its first port:

$$
\mathbf{T}_{1}=\left[\begin{array}{ccc}
\frac{9}{s+3} & -\frac{5}{s+1} & -\frac{2}{s+1} \\
-\frac{5}{s+1} & \frac{1}{s+3} & -\frac{53}{s+1} \\
-\frac{2}{s+1} & -\frac{53}{s+1} & \frac{2}{s+3}
\end{array}\right]
$$

According to Definition 3 and Property 4, we find that although the terminations $\frac{9}{s+3}, \frac{1}{s+3}$ and $\frac{2}{s+3}$ along each of the first three DOFs are passive, the coupled 3-DOF termination $\mathbf{T}_{1}$ is non-passive. Therefore, viewing the ter- mination impedances along each of the DOFs separately can result in misleading results in terms of absolute stability. As another case, consider a coupled 3-DOF bilateral teleoperator modeled as

$$
\left[\begin{array}{l}
F_{h} \\
F_{e}
\end{array}\right]=\mathcal{Z}\left[\begin{array}{l}
V_{h} \\
V_{e}
\end{array}\right]
$$

where $F_{h}=\left[f_{h x}, f_{h y}, f_{h z}\right]^{T}, F_{e}=\left[f_{e x}, f_{e y}, f_{e z}\right]^{T}, V_{h}=$ $\left[v_{h x}, v_{h y}, v_{h z}\right]^{T}, V_{e}=\left[v_{e x}, v_{e y}, v_{e z}\right]^{T}$, and

$$
\begin{aligned}
\mathcal{Z}= & {\left[\begin{array}{ll}
\mathbf{Z}_{11} & \mathbf{Z}_{12} \\
\mathbf{Z}_{21} & \mathbf{Z}_{22}
\end{array}\right] } \\
= & {\left[\begin{array}{llllll}
\frac{9}{s+3} & -\frac{5}{s+5} & -\frac{1}{s+5} & -\frac{1}{s+1} & -\frac{1}{s+2} & -\frac{2}{s+5} \\
\hdashline-\frac{5}{s+5} & \frac{9}{s+3} & -\frac{3}{s+5} & -\frac{1}{s+3} & -\frac{1}{s+1} & -\frac{1}{s+5} \\
-\frac{5}{s+5} & -\frac{3}{s+5} & \frac{9}{s+3} & -\frac{1}{s+3} & -\frac{1}{s+1} & -\frac{1}{s+1} \\
\hdashline-\frac{1}{s+1} & -\frac{1}{s+3} & -\frac{2}{s+3} & \frac{1}{s+3} & -\frac{9}{s+1} & -\frac{2}{s+3} \\
\hdashline-\frac{1}{s+2} & -\frac{1}{s+1} & -\frac{1}{s+3} & -\frac{9}{s+1} & \frac{1}{s+3} & -\frac{1}{s+3} \\
-\frac{1}{s+2} & -\frac{1}{s+3} & -\frac{1}{s+1} & -\frac{9}{s+1} & \frac{1}{s+3} & \frac{1}{s+3}
\end{array}\right] }
\end{aligned}
$$

Assume the terminations of this teleoperator are always passive. For using Llewellyn's criterion once along the $x$ direction, once along the $y$ direction, and once along the $z$ direction, we have to consider the following two subsystems of $(2)$ :

where

$$
\left[\begin{array}{c}
f_{h i} \\
f_{e i}
\end{array}\right]=\mathbf{Z}_{i}\left[\begin{array}{c}
v_{h i} \\
v_{e i}
\end{array}\right], \quad i=x, y, z
$$

$$
\mathbf{Z}_{x}=\mathbf{Z}_{y}=\mathbf{Z}_{z}=\left[\begin{array}{cc}
\frac{9}{s+3} & -\frac{1}{s+1} \\
-\frac{1}{s+1} & \frac{1}{s+3}
\end{array}\right]
$$

While the subsystems involving $\mathbf{Z}_{x}, \mathbf{Z}_{y}$, and $\mathbf{Z}_{z}$ always satisfy Llewellyn's criterion, as shown next, the coupled 3DOF teleoperator (2) is not absolutely stable. In general, for checking the absolute stability of a 3-port network such as a bilateral teleoperator, the port \#2 (environment port) can be connected to passive terminations while the input energy at the port \#1 (operator port) is measured. The bilateral teleoperator is absolutely stable if and only if, at all times $t>0$, we have Marquez (2003):

$$
E_{s}(t)=\int_{0}^{t} F_{h}^{T}(\tau) V_{h}(\tau) \mathrm{d} \tau \geq 0 .
$$

Similarly, the subsystems involving $\mathbf{Z}_{x}$ is absolutely stable if and only if, at all times $t>0$, we have

$$
E_{s}(t)=\int_{0}^{t} f_{h x}(\tau) v_{h x}(\tau) \mathrm{d} \tau \geq 0 .
$$

As shown in Figure 2, which plots $E_{s}(t)$ for the teleoperator (2) (solid) and each of the three subsystems (4) (dash-dot), simulations confirmed that each of the three 1-DOF subsystems are absolutely stable while the 3-DOF teleoperator is not absolutely stable (i.e., is potentially unstable). From the above examples, it is clear that for a 3-DOF haptic system, using Llewellyn's criterion thrice in each DOF is not useful as it ignores the coupling that may exist in the terminations and the teleoperator. To the best of our knowledge, no work has been done on direct absolute stability analysis of 3-DOF bilateral coupled teleoperators. 


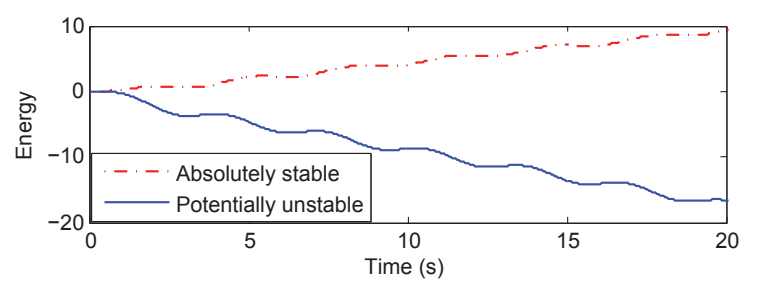

Fig. 2. Simulation results for analysis of absolute stability of the 3-DOF bilateral teleoperator (3). While the subsystems in (4) always satisfy Llewellyn's criterion as evidenced by the nonnegative energy plot (dashdot), the coupled 3-DOF teleoperator in (3) is actually potentially unstable as evidenced by the negative energy plot (solid).

Motivated by these facts, we propose a new absolute stability criterion of 3-DOF bilateral haptic systems.

\section{MATHEMATICAL PRELIMINARIES}

Notation 1. a is a scalar, $A$ is a vector, $\mathbf{A}$ is a matrix, and $\mathcal{A}$ is a block matrix (i.e., with matrix elements).

Definition 2. A multi-port network is passive if the total energy delivered to the network at its ports is nonnegative.

Definition 3. Khalil (2002) A $n \times n$ proper rational transfer matrix $\mathbf{G}(\mathbf{s})$ is positive real if

i) Poles of all elements of $\mathbf{G}(\mathbf{s})$ are in $\operatorname{Re}[s] \leq 0$,

ii) Any pure imaginary pole $j \omega$ of any element of $\mathbf{G}(\mathbf{s})$ is a simple pole and the residue matrix $\lim _{s \rightarrow j \omega}(s-j \omega) \mathbf{G}(\mathbf{s})$ is positive semidefinite Hermitian,

iii) For all real $\omega$ for which $j \omega$ is not a pole of any element of $\mathbf{G}(\mathbf{s})$, the matrix $\mathbf{G}(j \omega)+\mathbf{G}^{T}(-j \omega)$ is positive semidefinite.

Property 4. A Hermitian matrix, i.e., a square matrix equal to its conjugate transpose, is positive definite if its leading principal minors are all positive.

Lemma 5. Khalil (2002) A linear time-invariant minimal realization model with transfer matrix $\mathbf{G}(\mathbf{s})$ is passive if $\mathbf{G}(\mathbf{s})$ is positive real.

Definition 6. A multi-port network is absolutely stable if the coupled system remains bounded-input boundedoutput stable under all possible passive terminations. Otherwise, it is potentially unstable.

Lemma 7. Youla (1959) Let $\mathbf{Z}=\mathbf{Z}^{\mathrm{T}}$ be the impedance matrix of a reciprocal $n$-port network. Then, the network is passive if and only if it is absolutely stable.

Lemma 8. Youla (1960) Let $\mathbf{Z}_{\mathbf{1}}$ and $\mathbf{Z}_{\mathbf{2}}$ be the impedance matrices of two $n$-port networks. Then, if $\mathbf{Z}_{\mathbf{1}}$ and $\mathbf{Z}_{\mathbf{2}}$ possess identical principal minors of all orders, then $\mathbf{Z}_{\mathbf{1}}$ is absolutely stable if and only if $\mathbf{Z}_{\mathbf{2}}$ is absolutely stable.

\section{MAIN RESULT: AN ABSOLUTE STABILITY CRITERION FOR 3-DOF BILATERAL HAPTIC SYSTEMS}

An 3-DOF bilateral teleoperation system can be modeled as an 2-port network where each port (terminal) connects to an 3 -DOF termination as shown in Figure 1. The network impedance model will be

$$
\mathcal{F}=\mathcal{Z} \mathcal{V}
$$

where

$$
\begin{aligned}
& \mathcal{F}=\left[\begin{array}{ll}
F_{1} & F_{2}
\end{array}\right]^{T} \\
& \mathcal{V}=\left[\begin{array}{ll}
V_{1} & V_{2}
\end{array}\right]^{T}
\end{aligned}
$$

and $F_{i}$ and $V_{i}, i=1,2$, represent the $3 \times 1$ vectors of force and velocity at the $i^{\text {th }}$ port of the network, respectively. The impedance matrix of the network will be

$$
\mathcal{Z}=\left[\begin{array}{ll}
\mathbf{Z}_{11} & \mathbf{Z}_{12} \\
\mathbf{Z}_{21} & \mathbf{Z}_{22}
\end{array}\right]
$$

where $\mathbf{Z}_{i j}, i, j=1,2$, are $3 \times 3$ matrices given in (12).

$$
\mathbf{Z}_{i j}=\left[\begin{array}{ccc}
z_{3 i-2,3 j-2} & z_{3 i-2,3 j-1} & z_{3 i-2,3 j} \\
z_{3 i-1,3 j-2} & z_{3 i-1,3 j-1} & z_{3 i-1,3 j} \\
z_{3 i, 3 j-2} & z_{3 i, 3 j-1} & z_{3 i, 3 j}
\end{array}\right]
$$

On the other hand, the pair of 3-dimensional terminations are represented by

$$
\mathcal{T}=\operatorname{diag}\left[\mathbf{T}_{1}, \mathbf{T}_{2}\right]
$$

where $\mathbf{T}_{i}, i=1,2$, represents the $3 \times 3$ impedance matrix of the $i^{\text {th }} m$-dimensional termination.

Let

$$
\begin{aligned}
& \mathcal{Z}^{\prime}=\left[\begin{array}{cccc}
z_{1,1} & \gamma_{1} \sqrt{z_{1,2} z_{2,1}} & \cdots & \gamma_{5} \sqrt{z_{1,6} z_{6,1}} \\
\gamma_{1} \sqrt{z_{1,2} z_{2,1}} & z_{2,2} & \cdots & \gamma_{9} \sqrt{z_{2,6} z_{6,2}} \\
\vdots & \vdots & \cdots & \vdots \\
\gamma_{5} \sqrt{z_{1,6} z_{6,1}} & \gamma_{9} \sqrt{z_{2,6} z_{6,2}} & \cdots & z_{6,6}
\end{array}\right] \\
& \text { where, } \quad \gamma_{i}= \pm 1, \quad i=1,2, \cdots, 15
\end{aligned}
$$

Theorem 9. An 3-DOF bilateral haptic system with impedance matrix $\mathcal{Z}$ in (11) satisfying the symmetrization conditions

A) $z_{i, j} z_{j, k} z_{k, i}=z_{j, i} z_{k, j} z_{i, k}$, where $i, j, k=1,2, \cdots, 6$, $i \neq j \neq k$, and $i \neq k$.

B) $\mathbf{Z}_{\ell \ell}$ is symmetric, where $\ell=1,2$.

is absolutely stable if and only if

C) The elements of $\mathcal{Z}$ matrix in (12) have no poles in the right-half plane (RHP).

D) Any poles of the elements of the $\mathcal{Z}$ matrix in (12) on the imaginary axis are simple, and the leading principal minors of the residues matrix of the $\mathcal{Z}$ matrix at these poles are greater than zero.

E) For all real values of frequencies $\omega$, the leading principal minors of the real part of the $\mathcal{Z}^{\prime}$ matrix in (14) are greater than or equal zero, or equivalently

$$
\begin{aligned}
& \operatorname{Re}\left(z_{i, i}\right) \geq 0, \quad i=1,2, \cdots 6 \\
& \operatorname{Re}\left(z_{1,1}\right) \operatorname{Re}\left(z_{2,2}\right)-\frac{\left|z_{1,2} z_{2,1}\right|+\operatorname{Re}\left(z_{1,2} z_{2,1}\right)}{2} \geq 0
\end{aligned}
$$

$$
\operatorname{det}\left(\operatorname{Re}\left(\mathcal{Z}^{\prime}\right)\right) \geq 0
$$

Proof. Consider a linear time-invariant system with impulse response $h(t)$. The system's transfer function is the Laplace transform of $h(t)$ defined as

$$
H(s)=\int_{0}^{\infty} h(t) e^{-s t} d t
$$

where $s=\sigma+j \omega . H(s)$ is stable if every bounded input produces a bounded output and this happens if the poles 
of $H(s)$ have negative real parts. This stability definition is equivalent to the absolute convergence (defined below) of $H(s)$ in the region $\operatorname{Re}(s) \geq 0$. If $h$ is locally integrable, then $H(s)$ is said to converge if the limit $H(s)=\lim _{r \rightarrow \infty} \int_{0}^{r} h(t) e^{-s t} d t$ exists. Also, $H(s)$ is said to converge absolutely if the integral $\int_{0}^{\infty}\left|h(t) e^{-s t}\right| d t$ exists. The set of values of $s$ for which $H(s)$ converges is known as the region of convergence (ROC) and is of the form $\operatorname{Re}(s) \geq a$, where $a$ is a real constant. Importantly, if $H(s)$ converges at $s=s_{0}$, then it automatically converges for all $s$ with $\operatorname{Re}(s)>\operatorname{Re}\left(s_{0}\right)$. The above means that for stability analysis it suffices to focus on the convergence of $H(s)$ when $\operatorname{Re}(s)=0$, i.e., on the $j \omega$ axis. This is sometimes referred to as real-frequency stability. Thus, as a linear time-invariant system, the stability of an $m$-DOF $n$-lateral haptic system coupled to an $m$-DOF termination at each of its ports needs to only be analyzed for $s=j \omega$.

An $n$-port network is stable if the port currents $I_{1}, I_{2}, \cdots, I_{n}$ are zero under all passive terminations $t_{1}, t_{2}, \cdots, t_{n}$ for ports Youla (1959). In other words, an $n$-port network with an impedance matrix $Z_{n \times n}$ is stable if and only if the equation $\left(Z+T_{0}\right) I=0$, where $I=\left[I_{1}, I_{2}, \cdots, I_{n}\right]^{T}$ and $T_{0}=\operatorname{diag}\left[t_{1}, t_{2}, \cdots, t_{n}\right]$ has only the trivial solution $I=0$ for every passive choice of $T_{0}$; this happens if and only if $\operatorname{det}\left(Z+T_{0}\right) \neq 0$. On the other hand, according to Youla (1960), if two $n \times n$ matrices $Z_{1}$ and $Z_{2}$ have identical principal minors of all orders, then

$$
\operatorname{det}\left(Z_{1}+T_{0}\right)=\operatorname{det}\left(Z_{2}+T_{0}\right)
$$

for any $T_{0}=\operatorname{diag}\left[t_{1}, t_{2}, \cdots, t_{n}\right]$. This implies that the stability of two $n$-port networks with impedance matrices $Z_{1}$ and $Z_{2}$ will happen at the same time (Lemma 8).

Now, if there exists a reciprocal $n$-port network with impedance matrix $\mathcal{Z}^{\prime}$ that has the same stability characteristics as the original nonreciprocal $n$-port network with impedance matrix $\mathcal{Z}$, then

$$
\operatorname{det}\left(\mathcal{Z}^{\prime}+\mathcal{T}\right)=\operatorname{det}(\mathcal{Z}+\mathcal{T})
$$

for any passive $\mathcal{T}$ in (13). The above is to hold for any passive $\mathcal{T}$. It is easy to show that calculating the two determinants and equating the coefficients of $\mathbf{T}_{1}, \mathbf{T}_{2}$ gives the matrix $\mathcal{Z}^{\prime}$ in (14) as well as the symmetrization conditions $\mathrm{A}$ and $\mathrm{B}$.

On the other hand, according to Lemma 7, the reciprocal $n$-port network with impedance matrix $\mathcal{Z}^{\prime}$ is absolutely stable if and only if it is passive. In turn, according to Lemma $5, \mathcal{Z}^{\prime}$ is passive if and only if it is positive real which can be verified through Definition 3 .

From the above, we conclude that the original nonreciprocal $n$-port network with impedance matrix $\mathcal{Z}$ is absolutely stable if and only if the equivalent reciprocal $n$-port network's impedance matrix $\mathcal{Z}^{\prime}$ is positive real. In this context, it is straightforward to show that Conditions $\mathrm{C}$ and $\mathrm{D}$ in Theorem 9 are the same as Conditions i) and ii) in Definition 3. Also, according to Condition iii) of Definition 3, the Hermitian matrix

$$
\mathcal{Z}^{\prime}(j \omega)+\mathcal{Z}^{\prime T}(-j \omega)=2 \operatorname{Re}\left(\mathcal{Z}^{\prime}(j \omega)\right)
$$

needs to be positive semidefinite for the $n$-port network with impedance matrix $\mathcal{Z}$ to be absolutely stable. Using Property 4, and simplifying the conditions by

$$
\left(\operatorname{Re}\left(\sqrt{z_{i, j} z_{j, i}}\right)\right)=\sqrt{\frac{\left|z_{i, j} z_{j, i}\right|+\operatorname{Re}\left(z_{i, j} z_{j, i}\right)}{2}}
$$

where $i, j=1,2, \cdots, 6$, we arrive at conditions (15a)(15c). This concludes the proof.

\section{CASE STUDY: ABSOLUTE STABILITY OF A 3-DOF BILATERAL HAPTIC SYSTEM}

In this section, the aim is to apply the proposed absolute stability criterion to a coupled 3-DOF bilateral haptic teleoperation system. Then, simulations will be conducted for verifying the theoretical absolute stability conditions.

\subsection{A 3-DOF bilateral teleoperation system}

In a 3-DOF LTI bilateral teleoperation system, the dynamics of the master and the slave in contact with the user and the environment, respectively, are

$$
\begin{aligned}
\mathbf{Z}_{m} V_{h} & =F_{h}+F_{c m} \\
\mathbf{Z}_{s} V_{e} & =F_{e}+F_{c s}
\end{aligned}
$$

modeling each robot by a mass, where $\mathbf{Z}_{m}=\mathbf{M}_{m} s$, and $\mathbf{Z}_{s}=\mathbf{M}_{s} s$, are $3 \times 3$ impedance matrices of the master and the slave, respectively. Assume

$$
\mathbf{M}_{i}=\left[\begin{array}{lll}
m_{i x x} & m_{i x y} & m_{i x z} \\
m_{i x y} & m_{i y y} & m_{i y z} \\
m_{i x z} & m_{i y z} & m_{i z z}
\end{array}\right]
$$

where $i=m, s$ correspond to the master and the slave, respectively. Also, $F_{h}=\left[f_{h x}, f_{h y}, f_{h z}\right]^{T}$ denotes the interaction force vector between the user and the master and $F_{e}=\left[f_{e x}, f_{e y}, f_{e z}\right]^{T}$ denotes the interaction force vector between the slave and the environment. Lastly, $V_{h}=\left[v_{h x}, v_{h y}, v_{h z}\right]^{T}$ and $V_{e}=\left[v_{e x}, v_{e y}, v_{e z}\right]^{T}$ are the user and the environment velocities.

For simplicity, let us consider the position-position control laws as a special case Tavakoli et al. (2007):

$$
\begin{aligned}
F_{c m} & =-\mathbf{C}_{m} V_{h}+\mathbf{C}_{m} V_{e} \\
F_{c s} & =-\mathbf{C}_{s} V_{e}+\mathbf{C}_{s} V_{h}
\end{aligned}
$$

where the normally PD position controllers show up as PI velocity controllers:

$$
\begin{aligned}
\mathbf{C}_{m}= & {\left[\begin{array}{lll}
\frac{k_{p m x x}+k_{v m x x} s}{s} & \frac{k_{p m x y}+k_{v m x y} s}{s} & \frac{k_{p m x z}+k_{v m x z} s}{s} \\
\frac{k_{p m y x}+k_{v m y x} s}{s} & \frac{k_{p m y y}+k_{v m y y} s}{s} & \frac{k_{p m y z}+k_{v m y z} s}{s} \\
\frac{k_{p m z x}+k_{v m z x} s}{s} & \frac{k_{p m z y}+k_{v m z y} s}{s} & \frac{k_{p m z z}+k_{v m z z} s}{s}
\end{array}\right] } \\
\mathbf{C}_{s}= & {\left[\begin{array}{lll}
\frac{k_{p s x x}+k_{v s x x} s}{s} & \frac{k_{p s x y}+k_{v s x y} s}{s} & \frac{k_{p s x z}+k_{v s x z} s}{s} \\
\frac{k_{p s y x}+k_{v s y x} s}{s} & \frac{k_{p s y y}+k_{v s y y} s}{s} & \frac{k_{p s y z}+k_{v s y z} s}{s} \\
\frac{k_{p s z x}+k_{v s z x} s}{s} & \frac{k_{p s z y}+k_{v s z y} s}{s} & \frac{k_{p s z z}+k_{v s z z} s}{s}
\end{array}\right] }
\end{aligned}
$$

By substituting (23) in (21), the impedance matrix representation of the 3 -DOF teleoperator is found as

$$
\left[\begin{array}{c}
F_{h} \\
F_{e}
\end{array}\right]=\left[\begin{array}{cc}
\mathbf{C}_{m}+\mathbf{Z}_{m} & -\mathbf{C}_{m} \\
-\mathbf{C}_{s} & \mathbf{C}_{s}+\mathbf{Z}_{s}
\end{array}\right]\left[\begin{array}{l}
V_{h} \\
V_{e}
\end{array}\right]
$$

Now, let us investigate the absolute stability of the teleoperator via Theorem 9 for the case of $m=3$ and $n=3$. With $s=j \omega$, the symmetrization conditions of A and $\mathrm{B}$ boils down to the following 12 conditions involving the control gains and the frequency $\omega$ : 


$$
\begin{aligned}
& k_{v m y x}-k_{v m x y}+\frac{j\left(k_{p m x y}-k_{p m y x}\right)}{\omega}=0 \\
& k_{v m x z}-k_{v m z x}+\frac{j\left(k_{p m z x}-k_{p m x z}\right)}{\omega}=0 \\
& k_{v m y z}-k_{v m z y}+\frac{j\left(k_{p m z y}-k_{p m y z}\right)}{\omega}=0 \\
& k_{v s y x}-k_{v s x y}+\frac{j\left(k_{p s x y}-k_{p s y x}\right)}{\omega}=0 \\
& k_{v s x z}-k_{v s z x}+\frac{j\left(k_{p s z x}-k_{p s x z}\right)}{\omega}=0 \\
& k_{v s y z}-k_{v s z y}+\frac{j\left(k_{p s z y}-k_{p s y z}\right)}{\omega}=0 \\
& \omega^{2}\left(k_{v m y x} k_{v s y y}-k_{v s y x} k_{v m y y}\right)+j \omega\left(k_{v s y x} k_{p m y y}\right. \\
& \left.+k_{p s y x} k_{v m y y}-k_{v m y x} k_{p s y y}-k_{p m y x} k_{v s y y}\right) \\
& +k_{p s y x} k_{p m y y}-k_{p m y x} k_{p s y y}=0 \\
& \omega^{2}\left(k_{v m x x} k_{v s y x}-k_{v s x x} k_{v m y x}\right)+j \omega\left(k_{v s x x} k_{p m y x}\right. \\
& \left.+k_{p s x x} k_{v m y x}-k_{v m x x} k_{p s y x}-k_{p m x x} k_{v s y x}\right) \\
& +k_{p s x x} k_{p m y x}-k_{p m x x} k_{p s y x}=0 \\
& \omega^{2}\left(k_{v m x z} k_{v s x x}-k_{v s x z} k_{v m x x}\right)+j \omega\left(k_{v s x z} k_{p m x x}\right. \\
& \left.+k_{p s x z} k_{v m x x}-k_{v m x z} k_{p s x x}-k_{p m x z} k_{v s x x}\right) \\
& +k_{p s x z} k_{p m x x}-k_{p m x z} k_{p s x x}=0 \\
& \omega^{2}\left(k_{v m z z} k_{v s x z}-k_{v s z z} k_{v m x z}\right)+j \omega\left(k_{v s z z} k_{p m x z}\right. \\
& \left.+k_{p s z z} k_{v m x z}-k_{v m z z} k_{p s x z}-k_{p m z z} k_{v s x z}\right) \\
& +k_{p s z z} k_{p m x z}-k_{p m z z} k_{p s x z}=0 \\
& +\omega^{2}\left(k_{v m y z} k_{v s y y}-k_{v s y z} k_{v m y y}\right)+j \omega\left(k_{v s y z} k_{p m y y}\right. \\
& \left.+k_{p s y z} k_{v m y y}-k_{v m y z} k_{p s y y}-k_{p m y z} k_{v s y y}\right) \\
& +k_{p s y z} k_{p m y y}-k_{p m y z} k_{p s y y}=0 \\
& \left.+k_{p s z z z} k_{v m y z}-k_{v m z z} k_{p s y z}-k_{p m z z} k_{v s y z}\right) \\
& +k_{p s z z} k_{p m y z}-k_{p m z z} k_{p s y z}=0 \\
& +3 k_{v y z} \\
& +3
\end{aligned}
$$

Conditions (26)-(37) will be fulfilled for all frequencies $\omega$ if the gains of the PD controllers (24) satisfy

$k_{p m x y}=k_{p m y x}, \quad k_{v m y z}=k_{v m z y}, \quad k_{v m x z}=k_{v m z x}$

\begin{tabular}{|c|c|c|c|c|c|c|}
\hline \multirow{4}{*}{ Master } & $k_{p m x x}$ & 40 & $k_{\text {pmyy }}$ & 40 & $k_{p m z z}$ & 25 \\
\hline & $k_{v m x x}$ & 80 & $k_{v m y y}$ & 80 & $k_{v m z z}$ & 50 \\
\hline & $k_{p m x y}$ & 4 & $k_{p m x z}$ & 3 & $k_{p m y z}$ & 30 \\
\hline & $k_{v m x y}$ & 8 & $k_{v m x z}$ & 6 & $k_{v m y z}$ & $60(6)$ \\
\hline \multirow{4}{*}{ Slave } & $k_{p s x x}$ & 80 & $k_{\text {psyy }}$ & 80 & $k_{p s z z}$ & 50 \\
\hline & $k_{v s x x}$ & 160 & $k_{v s y y}$ & 160 & $k_{v s z z}$ & 100 \\
\hline & $k_{p s x y}$ & 8 & $k_{p s x z}$ & 6 & $k_{\text {psyz }}$ & 60 \\
\hline & $k_{v s x y}$ & 16 & $k_{v s x z}$ & 12 & $k_{v s y z}$ & 120 \\
\hline
\end{tabular}

$k_{p s x y}=k_{p s y x}, \quad k_{v s y z}=k_{v s z y}, \quad k_{v s x z}=k_{v s z x}$

$\frac{k_{v m x x}}{k_{v s x x}}=\frac{k_{v m x y}}{k_{v s x y}}=\frac{k_{v m x z}}{k_{v s x z}}=\frac{k_{v m y y}}{k_{v s y y}}=\frac{k_{v m y z}}{k_{v s y z}}=\frac{k_{v m z z}}{k_{v s z z}}$

$=\frac{k_{p m x x}}{k_{p s x x}}=\frac{k_{p m x y}}{k_{p s x y}}=\frac{k_{p m x z}}{k_{p s x z}}=\frac{k_{p m y y}}{k_{p s y y}}=\frac{k_{p m y z}}{k_{p s y z}}=\frac{k_{p m z z}}{k_{p s z z}}$

(38c)

It is easy to see that, under (38), all the elements of the impedance matrix (25) have only a simple pole on the imaginary axis, thus satisfying Condition C. Analysis of the residues according to Condition D leads to the following constraints:

$$
\begin{aligned}
& k_{p m x x} \geq 0, \quad k_{p m y y} \geq 0, \quad k_{p m z z} \geq 0 \\
& k_{p s x x} \geq 0, \quad k_{p s y y} \geq 0, \quad k_{p s z z} \geq 0 \\
& k_{p m x x} k_{p m y y}-k_{p m x y}^{2} \geq 0 \\
& k_{p m x x} k_{p m y y} k_{p m z z}-k_{p m x x} k_{p m y z}^{2}-k_{p m y y} k_{p m x z}^{2} \\
& -k_{p m z z} k_{p m x y}^{2}+2 k_{p m x y} k_{p m x z} k_{p m y z} \geq 0
\end{aligned}
$$

It is easy to see that, the condition set (39) holds if

$$
k_{p m x x} k_{p m y y} \geq k_{p m x y}^{2}
$$

and condition (39d) hold.
Table 1. The controllers gains of the $3-\mathrm{DOF}$ bilateral teleoperation system used in experiments.

Now, let us deal with Condition E of Theorem 9. Condition (15a) turns out to state

$$
\begin{aligned}
& k_{v m x x} \geq 0, \quad k_{v m y y} \geq 0, \quad k_{v m z z} \geq 0 \\
& k_{v s x x} \geq 0, \quad k_{v s y y} \geq 0, \quad k_{v s z z} \geq 0
\end{aligned}
$$

Under (38) and (40), the second leading principal minor condition, i.e., (15b), gives

$$
k_{v m x x} k_{v m y y}-k_{v m x y}^{2} \geq 0
$$

Similarly, the third leading principal minor condition requires

$$
\begin{aligned}
& k_{v m x x} k_{v m y y} k_{v m z z}-k_{v m x x} k_{v m y z}^{2}-k_{v m y y} k_{v m x z}^{2} \\
& -k_{v m z z} k_{v m x y}^{2}+2 k_{v m x y} k_{v m x z} k_{v m y z} \geq 0
\end{aligned}
$$

The fourth leading principal minor condition mandates

$$
\begin{aligned}
& -K_{v m x z}^{2}\left(k_{v m x x} k_{v m y y}-k_{v m y z}^{2}\right)\left(k_{v m z z}\right. \\
& \left.+k_{v s z z}+\sqrt{k_{v m z z} k_{v s z z}}\right) \geq 0
\end{aligned}
$$

Condition (44) will be fulfilled if the PD control gain

$$
k_{v m y z}^{2} \geq k_{v m x x} k_{v m y y}
$$

Finally, under above stability conditions, the fifth leading principal minor condition and the sixth leading principal minor condition, i.e., (15c) always greater than zero.

So, a sufficient, frequency-independent, and compact condition set for absolute stability of the above-described 3DOF bilateral teleoperator is

$$
\begin{aligned}
& k_{p m x y}=k_{p m y x}, \quad k_{v m y z}=k_{v m z y}, \quad k_{v m x z}=k_{v m z x} \\
& k_{p s x y}=k_{p s y x}, \quad k_{v s y z}=k_{v s z y}, \quad k_{v s x z}=k_{v s z x} \\
& \frac{k_{v m x x}}{k_{v s x x}}=\frac{k_{v m x y}}{k_{v s x y}}=\frac{k_{v m y y}}{k_{v s y y}}=\frac{k_{v m y z}}{k_{v s y z}}=\frac{k_{v m z z}}{k_{v s z z}}=\frac{k_{p m x x}}{k_{p s x x}} \\
& =\frac{k_{p m x y}}{k_{p s x y}}=\frac{k_{p m x z}}{k_{p s x z}}=\frac{k_{p m y y}}{k_{p s y y}}=\frac{k_{p m y z}}{k_{p s y z}}=\frac{k_{p m z z}}{k_{p s z z}} \\
& k_{p m x x} k_{p m y y} \geq k_{p m x y}^{2}, \quad k_{v m x x} k_{v m y y} \geq k_{v m x y}^{2} \\
& k_{v m x x} k_{v m y y} k_{v m z z}-k_{v m x x} k_{v m y z}^{2}-k_{v m y y} k_{v m x z}^{2} \\
& -k_{v m z z} k_{v m x y}^{2}+2 k_{v m x y} k_{v m x z} k_{v m y z} \geq 0 \\
& k_{v m y z}^{2} \geq k_{v m x x} k_{v m y y}
\end{aligned}
$$

where all control gains are nonnegative. Again, the ratios in (46) are merely artifacts of our presentation of the absolute stability conditions meaning that division by zero can be avoided.

\subsection{Simulations}

The position-position 3-DOF bilateral teleoperation system has been simulated in MATLAB/Simulink. There is no time delay in the communication channel between the masters and the slave. Two 3-DOF robots as the master and the slave are modeled by $M_{m x x}=1.7, M_{m x y}=0.2$, 


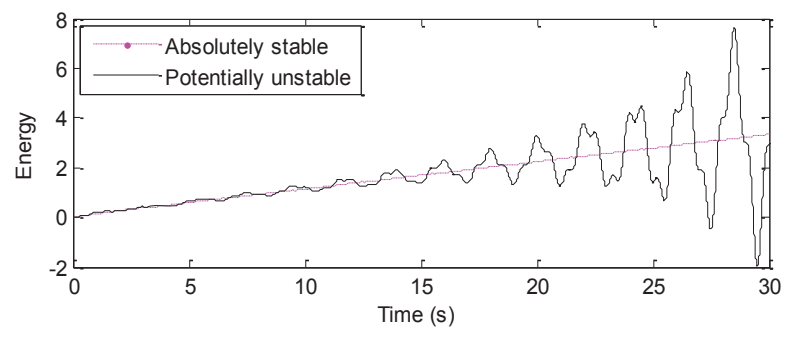

Fig. 3. Simulation results for the 3-DOF bilateral teleoperation system. Input energy at the master's port is shown while the slave is connected to LTI passive terminations. The control gains are listed in Table 1 for the stable case with $K_{v m y z}=60$ and for the potentially unstable case with $K_{v m y z}=6$.

$M_{m y y}=1.9, M_{m x z}=0.1, M_{m y z}=0.3, M_{m z z}=1.3$ $M_{s x x}=1.3, M_{s x y}=0.5, M_{s y y}=1.6, M_{s x z}=0.1$ $M_{s y z}=0.3$, and $M_{s z z}=1.5$, respectively.

According to (46), the stability of the position-position 3DOF bilateral teleoperation system should depend on the controllers gains. In the simulations, the controllers gains were chosen according to Table 1 .

In simulations, to check the absolute stability of the network, the slave ports is connected to LTI terminations

$$
\mathbf{T}_{1}=\left[\begin{array}{ccc}
\frac{8}{s+3} & -\frac{2}{s+1} & -\frac{1}{s+1} \\
-\frac{2}{s+1} & \frac{9}{s+3} & \frac{2}{s+1} \\
-\frac{1}{s+1} & \frac{2}{s+1} & \frac{5}{s+1}
\end{array}\right]
$$

which are passive. Port 1 is open and three sine-wave input $f_{h x}, f_{h y}, f_{h z}$ are applied to the master. The input energy $E_{s}(t)$ in (6) is plotted in Figure 3. As it can be seen, if the control gains are selected according to (46), e.g., as listed in Table 1, with $K_{v m y z}=60$, then the input energy at port 1 is non-negative at all times, indicating the absolute stability of the bilateral teleoperator. However, when we change $K_{v m y z}$ to 6 , which violate (46), the input energy $E_{s}(t)$ will become negative at least for a period of time, indicating potential instability of the bilateral teleoperator. The above show that there is agreement between the theoretical absolute stability condition (46) and the simulations.

\section{CONCLUSIONS}

In the beginning of this paper, it was shown via an example that applying Llewellyn's absolute stability criterion once in each DOF of a 3-DOF bilateral haptic system cannot guarantee the absolute stability as this method ignores the coupling between DOFs that may exist in the system.

This paper presented a closed-form and easy-to-use absolute stability criterion for 3-DOF bilateral haptic systems. Through a case studies, we elaborated on its application in absolute stability analysis of a 3-DOF bilateral haptic system. Through simulations, the proposed absolute stability criterion was validated.

\section{ACKNOWLEDGMENT}

This research was supported by the Natural Sciences and Engineering Research Council (NSERC) of Canada, by the
Natural Science Foundation of China (NFSC, Grant No. 51277022), and by the China Scholarship Council (CSC) under grant [2011]3005.

\section{REFERENCES}

Haykin, S. (1970). Active Network Theory. AddisonWesley.

Khademian, B. and Hashtrudi-Zaad, K. (2011). Shared control architectures for haptic training: Performance and coupled stability analysis. The International Journal of Robotics Research, 30, 1627-1642.

Khalil, H.K. (2002). Nonlinear Systems. Prentice Hall.

$\mathrm{Ku}, \mathrm{W}$. (1963). Stability of linear active nonreciprocal n-ports. J. Franklin Inst., (276), $207-224$.

Li, J., Tavakoli, M., and Huang, Q. (2013a). Stability analysis of trilateral haptic collaboration. IEEE World Haptics Conference 2013, $611-616$.

Li, J., Tavakoli, M., Mendez, V., and Huang, Q. (2013b). Conservatism of passivity criteria for stability analysis of trilateral haptic systems. IEEE World Haptics Conference 2013, $633-638$.

Llewellyn, F. (1952). Some fundamental properties of transmission systems. Proceedings of the IRE, 2(1), 271283.

Malysz, P. and Sirouspour, S. (2011). Trilateral teleoperation control of kinematically redundant robotic manipulators. International Journal of Robotics Research, 30, 1643-1664.

Marquez, H.J. (2003). Nonlinear Control Systems Analysis and Design. Wiley.

Mendez, V. and Tavakoli, M. (2010). A passivity criterion for n-port multilateral haptic systems. In 2010 49th IEEE Conference on Decision and Control (CDC), 274 $-279$.

Panzirsch, M., Artigas, J., Tobergte, A., Kotyczka, P., Carsten, P., Albu-Schaeffer, A., and Hirzinger, G. (2012). A peer-to-peer trilateral passivity control for delayed collaborative teleoperation. In Institute of Robotics and Mechatronics, volume 7282, 395-406.

Raisbeck, G. (1954). A definition of passive linear networks in terms of time and energy. Journal of Applied Physics, 25(12), 1510-1514.

Shahbazi, M., Talebi, H.A., and Yazdanpanah, M.J. (2010). A control architecture for dual user teleoperation with unknown time delays: A sliding mode approach. In 2010 IEEE/ASME International Conference on $A I M, 1221-1226$.

Speich, J. and Goldfarb, M. (2005). An implementation of loop-shaping compensation for multidegree-of-freedom macro-microscaled telemanipulation. IEEE Transactions on Control Systems Technology, 13(3), 459 - 464.

Tavakoli, M., Aziminejad, A., Patel, R., and Moallem, M. (2007). High-fidelity bilateral teleoperation systems and the effect of multimodal haptics. IEEE Transactions on Systems, Man, and Cybernetics, Part B: Cybernetics, $37(6), 1512-1528$.

Youla, D. (1959). A stability characterization of the reciprocal linear passive n-port. Proc. IRE, 47, 11501151.

Youla, D. (1960). A note on the stability of linear, nonreciprocal n-port. Proc. IRE, 48, 121-122. 